\title{
Creating the Deep Space Environment for Testing the James Webb Space Telescope at the Johnson Space Center's Chamber A
}

\author{
Jonathan L. Homan ${ }^{1}$, Mary P. Cerimele ${ }^{2}$, and Michael E. Montz ${ }^{3}$ \\ NASA Johnson Space Center, Houston, Texas, 77058, USA
}

\begin{abstract}
Chamber A is the largest thermal vacuum chamber at the Johnson Space Center and is one of the largest space environment chambers in the world. The chamber is $19.8 \mathrm{~m}(65 \mathrm{ft})$ in diameter and $36.6 \mathrm{~m} \mathrm{(120} \mathrm{ft)}$ tall and is equipped with cryogenic liquid nitrogen panels (shrouds) and gaseous helium shrouds to create a simulated space environment. It was originally designed and built in the mid 1960's to test the Apollo Command and Service Module and several manned tests were conducted on that spacecraft, contributing to the success of the program. The chamber has been used since that time to test spacecraft active thermal control systems, Shuttle DTO, DOD, and ESA hardware in simulated Low Earth Orbit (LEO) conditions. NASA is now moving from LEO towards exploration of locations with environments approaching those of deep space. Therefore, Chamber A has undergone major modifications to enable it to simulate these deeper space environments. Environmental requirements were driven, and the modifications were funded, by the James Webb Space Telescope program, and this telescope which will orbit Solar/Earth L2, will be the first test article to benefit from the chamber's new capabilities. To accommodate JWST, the Chamber A high vacuum system has been modernized, additional LN2 shrouds have been installed, the liquid nitrogen system has been modified to remove dependency on electrical power and increase its reliability, a new helium shroud/refrigeration system has been installed to create a colder more stable and uniform heat sink and, the controls have been updated to increase the level of automation and improve operator interfaces.

Testing of these major modifications was conducted in August 2012 and this initial test was very successful, with all major systems exceeding their performance requirements. This paper will outline the changes in the overall environmental requirements, discuss the technical design data that was used in the decisions leading to the extensive modifications, and describe the new capabilities of the chamber.
\end{abstract}

\footnotetext{
${ }^{1}$ Senior Engineer, Systems Test Branch, CTSD/EC4

2 JSC-JWST Project Manager, Systems Test Branch, CTSD, JSC/EC4, AIAA Senior Member

${ }^{3}$ Deputy Branch Chief, Systems Test Branch, CTSD, JSC/EC4
}

1

American Institute of Aeronautics and Astronautics 